\title{
Marathons and myasthenia gravis: a case report
}

Simone Birnbaum ${ }^{1,2,3^{*}}$ (D), Tarek Sharshar ${ }^{4,5,6}$, Bruno Eymard ${ }^{1}$, Marie Theaudin' ${ }^{7}$, Pierre Portero ${ }^{2}$ and Jean-Yves Hogrel ${ }^{1}$

\begin{abstract}
Background: The cardinal symptoms of auto-immune myasthenia gravis are fatigue and weakness. Endurance events such as marathon running would seem incompatible with this chronic disease. Many patients stop sport altogether. There is limited literature of patients with auto-immune myasthenia gravis undergoing regular endurance exercise.

Case presentation: We report the case of a 36-year-old female who began long-distance running whilst experiencing initial symptoms of myasthenia gravis. She was diagnosed with auto-immune myasthenia gravis and whilst advised to stop all sport, her way of fighting and living with this chronic and unpredictable disease was to continue running to maintain a healthy body and mind. Despite suffering from ocular, bulbar and localized limb fatigability, she managed to complete multiple marathons and achieve disease stability with cholinesterase inhibitors.

Conclusions: Marathon and half-marathon running lead to distinct changes in mediators of inflammation in an exercise-dose-dependent manner. Despite symptoms of weakness and fatigue in certain muscles in myasthenia gravis, physical exertion remains possible and may not worsen symptoms as demonstrated in this case and recent studies. The immunomodulatory role of exercise could be considered in this case however this hypothesis remains to be confirmed in future studies with quantitative data.
\end{abstract}

Keywords: Myasthenia gravis, Marathon, Auto-immune disease, Health, Endurance exercise

\section{Background}

Auto-immune myasthenia gravis (MG) is a chronic disease whereby dysfunction at the neuromuscular junction causes symptoms of fatigue and weakness [1]. Endurance events such as marathon running would seem incompatible with MG. Many patients stop sport altogether, finding activities of daily living to be challenging enough in themselves [2]. Even for the general public, marathon running is not practiced by a large majority, one must train regularly and have a high endurance capacity.

There are only three case studies combining sport and MG reported in the literature. Scheer et al. (2012) describe a 52 year old ultra-endurance athlete with mild MG (MGFA IIa), treated with $10 \mathrm{mg}$ of prednisone who completed a $220 \mathrm{~km}$ ultra-marathon over 5 days [3].

\footnotetext{
* Correspondence: s.birnbaum@institut-myologie.org

${ }^{1}$ Institute of Myology, GH Pitié-Salpêtrière (AP-HP), Bd de l'Hôpital, 75651

Paris Cedex 13, France

${ }^{2}$ Bioingénierie, Tissus et Neuroplasticité, EA 7377 Université Paris-Est Créteil

Faculté de Médecine, 8 rue Jean Sarrail, 94010 Créteil, France

Full list of author information is available at the end of the article
}

Fatigue, leg weakness, dysphagia and breathing problems were reported, relieved by rest, shade and $60 \mathrm{mg}$ of pyridostigmine every $90 \mathrm{~min}$ ( $\max$ daily dose $720 \mathrm{mg}$ ). He began running 5 years prior to MG diagnosis and maintained a combined weekly running distance of $70 \mathrm{~km}$. Stout et al. (2001) report a 26 year old athletic (baseball and weight lifting) student who suffered from extreme weakness and atrophy due to his MG, he was unable to do a single push-up at diagnosis [4]. Once stabilized he began exercising again at low levels which prevented further weakness but he struggled to regain force until undergoing a 15-wk upper and lower limb resistance training program, with oral creatine supplementation and he was able to make force gains (37\% leg extension) and increase training volume (34-40\% upper limb). Finally, Leddy et al. (2000) describe a 17 year old college football player with mild generalized MG (antibody-negative, 15\% decrement RNS-EMG), treated with $60 \mathrm{mg}$ prednisone every other day and $60 \mathrm{mg}$ pyridostigmine q.i.d [5]. Following an initial period of weakness at the time of diagnosis, his strength returned to normal after

(c) The Author(s). 2018 Open Access This article is distributed under the terms of the Creative Commons Attribution 4.0 International License (http://creativecommons.org/licenses/by/4.0/), which permits unrestricted use, distribution, and 
6 weeks and aside from a relapse associated with a period of non-compliance with medication, he became stable without treatment and was able to participate in full football practice.

Three recent uncontrolled trials have demonstrated benefits and tolerance of supervised physical activity (resistance or aerobic) programs for patients with stable MG [6-8]. Additionally, using the contralateral limb as a control, Lohi et al. found improvements in lower limb force following dynamic training and no deterioration nor negative side effects in a group of 11 subjects with mild MG [9]. A review article has suggested that risk factors for exacerbation of MG include prolonged exercise, running uphill and activity with stairs, however no specific data was provided to support these claims [10]. Currently there are no official guidelines regarding participation in sport and MG [11, 12].

\section{Case presentation}

Here we report a 36-year-old female nurse, working full-time 12-h night shifts in a busy intensive care service, with generalized (MGFA IIb) auto-immune MG, symptomatic and dependent on cholinesterase inhibitors. Past medical history includes eczema in her teens, she is a carrier for sickle disease (sickle cell trait (SCT)) and she carried two pregnancies to term. Red blood cells have slightly reduced mean corpuscular volume and mean corpuscular hemoglobin concentration, $75.5 \mathrm{fl}$ and $25.7 \mathrm{pg}$, respectively. She has no clinical symptoms of anemia and no specific treatment or monitoring. Surgical history includes 1 cesarean (2004), linea alba repair (2011) and breast implant (2014). She is a nonsmoker and does not drink alcohol. She is right-handed. She has a normal body mass index, $20.3 \mathrm{~kg} / \mathrm{m}^{2}$, weighing $52 \mathrm{~kg}$ for $1.60 \mathrm{~m}$.

Despite already experiencing abnormal weakness, she began running a year before being diagnosed with MG. Prior to running, she played amateur level basketball however this became incompatible with working night-shifts. MG diagnosis was based on clinical signs (right hand weakness - difficulty brushing teeth and hair, carrying light loads, cutting meat, a heavy head, nasal voice, ptosis, diplopia, dysphagia and difficulty masticating and articulating), serum auto-antibodies against nicotinic acetylcholine receptors (AChR) $(>100 \mathrm{nmol} / \mathrm{l})$ and significant decrement on repetitive nerve stimulation (3 Hz) EMG (50\% right trapezius, 24\% right anconeus, $15 \%$ left anconeus, $43 \%$ tongue/mouth (CN V/XII)). Myasthenic muscle score (MMS) was 65/100. Initial treatment consisted of intravenous immunoglobulins $(2 \mathrm{~g} / \mathrm{kg}$ over 3 days $(100 \mathrm{~g}))$ and $60 \mathrm{mg}$ pyridostigmine (t.i.d). Thoracic CT scan did not show thymoma but was in favour of thymic hyperplasia. Thymectomy was not performed as per the patient's request.

Despite the patient being informed at diagnosis that sport was contra-indicated, she kept running. Training consisted of 1-2 $10 \mathrm{~km}$ weekly runs with the beginning being the most difficult. MG symptoms persisted including fatigue, dysphagia and episodes of diplopia at the end of pyridostigmine dose thus aziathoprine (100 mg) was introduced. She performed a half marathon 2 months later, followed by a full marathon and another half marathon. No major difficulty was experienced and performances significantly improved: $5 \mathrm{~h} 13 \mathrm{mins}(8.1 \mathrm{~km} /$ h) for a marathon pre-diagnosis to $4 \mathrm{~h} 51 \mathrm{mins}(8.7 \mathrm{~km} / \mathrm{h})$ post diagnosis (and treatment), Figs. $1 \& 2$.

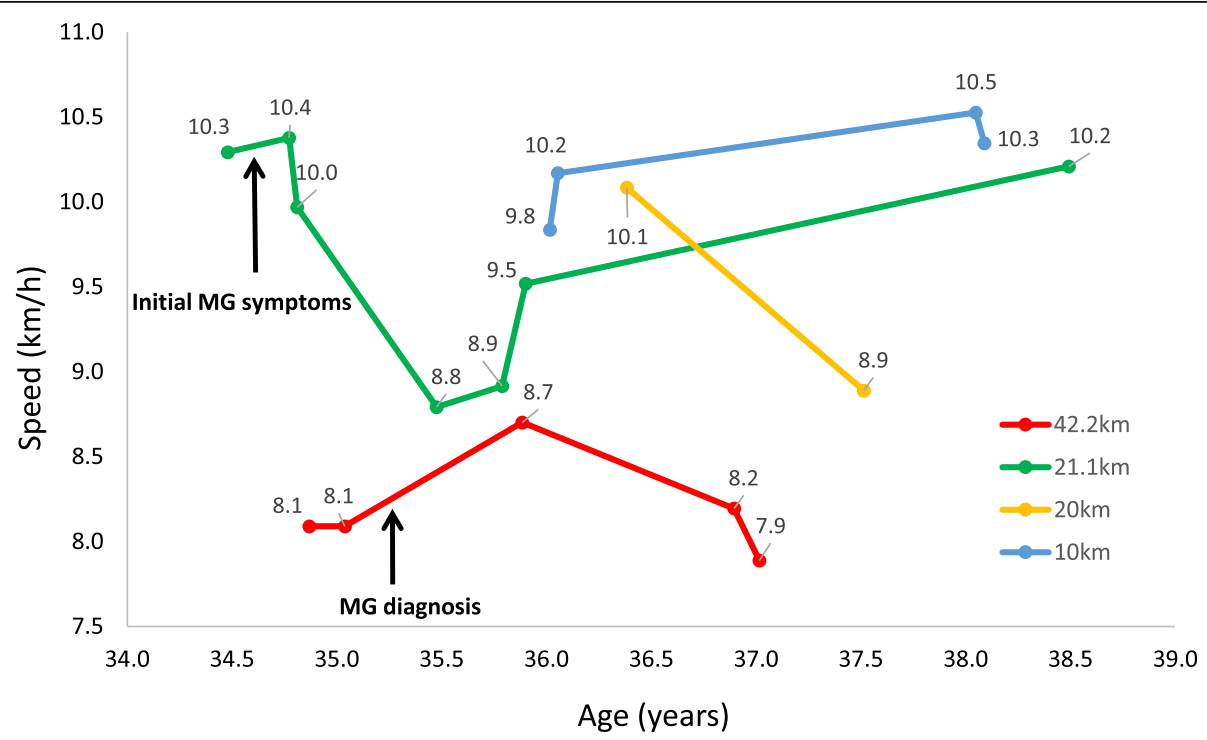

Fig. 1 Running performance timeline 


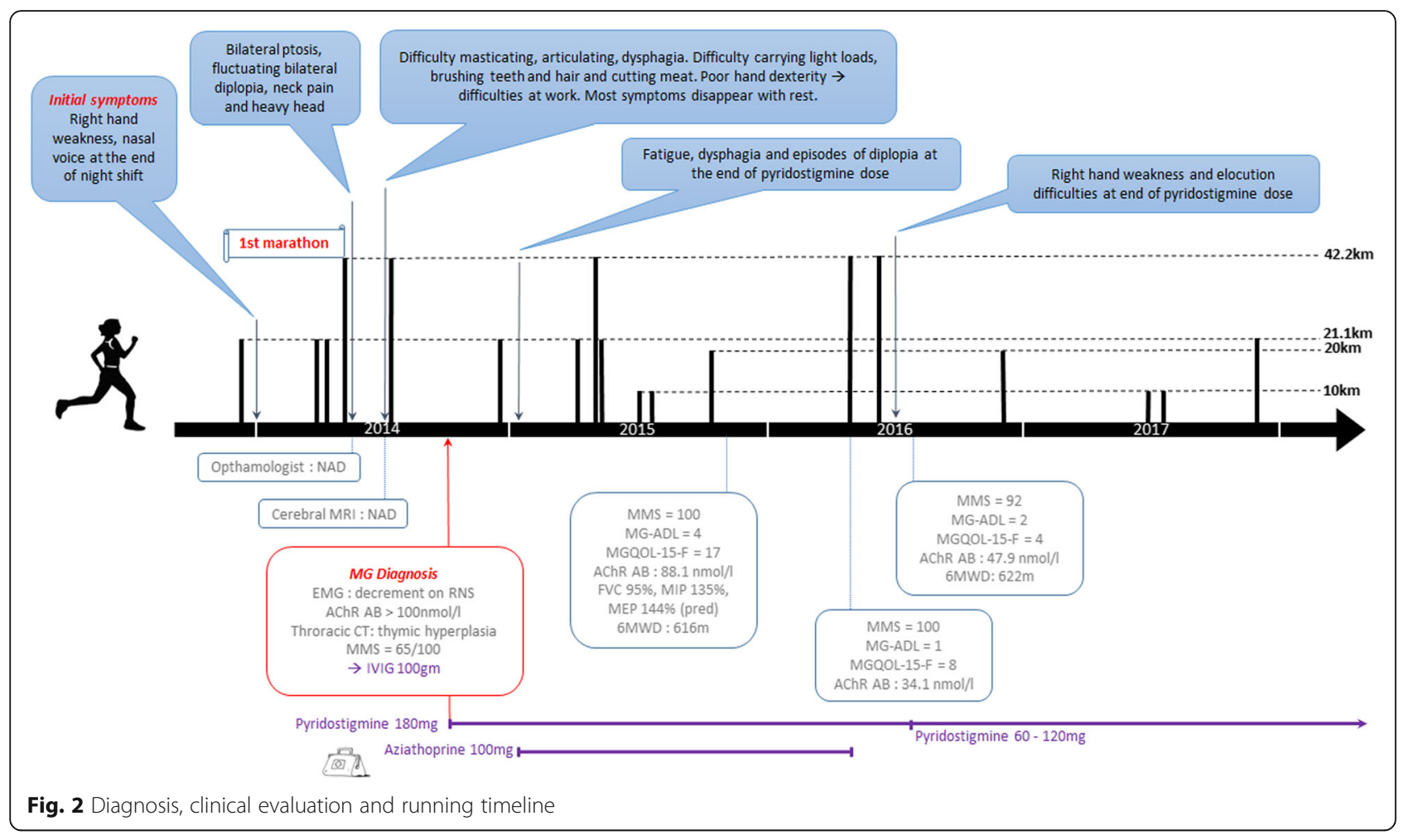

On clinical evaluation 1 year after MG diagnosis, she had above average respiratory strength (MIP and MEP $135 \%$ and $144 \%$ of theoretical) and normal respiratory function (FVC 95\% of theoretical). On maximal voluntary testing, limb strength was normal (knee extensors and elbow flexors: $128 \%$ and $101 \%$ of theoretical, respectively and right handgrip strength: $88 \%)$. Walking endurance was also considered normal (92\% of theoretical 6MWD). The MMS suffered from a ceiling effect with the patient achieving the maximal score of 100 and a score of 4 on the MG-ADL due to episodes of dysphagia, dyspnea with effort and UL fatigue with brushing her teeth and hair. She reported recurrent MG symptoms including loss of hand dexterity (difficulty manipulating medication at work), bulbar symptoms such as dysphagia on her own saliva, a nasal voice and ocular symptoms (ptosis and diplopia). MG-specific quality of life (MGQOL-15-F) was reduced (17/60) particularly with regards to professional and social aspects, reflected in the WHO-QOL Bref with the social relationship and physical health domains being reduced, $11 / 20$ and 13/20 respectively $[13,14]$.

She continued regular running without any particular changes in her MG, recurrent right hand weakness persisted and she experienced occasional ocular and bulbar symptoms primarily at the end of pyrodistigmine dose. Quality of life improved significantly over time (Fig. 2) and strength remained stable (knee extensors and elbow flexors: $128 \%$ and $100 \%$ of theoretical, respectively, right handgrip strength: 93\%). She discontinued aziathoprine (treatment duration 14 months) and remained exclusively treated with cholinesterase inhibitors $(60 \mathrm{mg}$, b.i.d).

\section{Discussion and conclusion}

It is well known that MG does not affect all muscles to the same extent and whilst some muscles may clearly be affected, other muscles may function normally and can be trained without adverse effects. Thus, continuing activities and daily life seems important for maintaining "unaffected" muscles and for psychological benefits. With regards to physical activity a close link between endurance running and the immune system activity has been demonstrated [15]. During sustained physical activity, repeated muscle contractions stimulate the production of inflammatory cytokines such as Interleukin-6 (IL-6) by myocytes. IL-6 may act as an inhibitor to pro-inflammatory cytokines such as TNF- $\alpha$ which is a potent mediator of tissue damage $[16,17]$. IL-6 also stimulates the production of IL-10 (an anti-inflammatory cytokine) and IL-1ra, generating an anti-inflammatory environment. Whether these effects can modify disease activity in MG currently remains unclear. These exercise-induced mediators of inflammation are susceptible to exercise dose, with greater increases seen following a marathon as compared to a half marathon, for example [16]. A disease-specific response has been suggested for the change in cytokine kinetics in relation to exercise [18]. 
Whilst evidence of exercise-induced change of cytokines remains sparse in MG, there is growing evidence of the presence of inflammation and modulation of the immune response by cytokines in MG pathogenesis [19, 20]. Certain cytokines are upregulated (IL-17, IL-21, IL-6, IL-10) and others downregulated (IL-4) compared to control subjects [19, 21-23]. A recent study evaluated serum IL-6 before and after a twice-weekly, 3 month training program in subjects with ocular or mild, generalized MG. Serum Il-6 levels remained unchanged [7]. However, circulating microRNAs (miR150-5p and miR21-5p) decreased [7]. These immuno-microRNAs have been suggested as new biomarkers in AChR MG, notably miR150-5p as a marker for disease severity $[24,25]$.

Another factor which warrants consideration here is the localized role of IL- 6 in triggering satellite cells in the myogenic process [26]. Whilst research has demonstrated the role of IL-6 in muscle atrophy, this has predominantly involved pathological situations such as cancer cachexia and ageing [27] where there is chronic inflammation. When released at low concentration into satellite cell niches IL- 6 could promote repair and regenerate skeletal muscle tissue [27]. It seems that IL-6 is deregulated in the muscles of MG patients, possibly due to AChR antibodies deregulating the IL-6 pathway [28].

In the case of this patient, weakness induced by MG mechanisms tends to affect the same muscles which were initially affected, regardless of increased exertion. Whether this patient has benefitted from protective effects of exercise or not is difficult to determine, however, this case study demonstrates that an endurance sport such as running marathons and MG are not mutually exclusive. The immunosuppressor treatment likely played an essential role in attenuating the autoimmune attack enabling the patient to improve and optimize her physical capacities. Clinical scores improved and remained stable and running performance improved and remained relatively consistent (Figs. 1 and 2). The level of antibodies against AChR decreased over time (Fig. 2) however this also coincided with immunosuppressor treatment.

In stabilized MG there are no clear arguments to fear adverse effects of physical activity and as well as positive psychological effects, patients will benefit from cardiovascular and muscular adaptions as well as possible immunomodulatory effects. It is important to consider each individual case and weigh up the benefits and risks and possibly put into place a supervised or specific return to sport program where necessary. As can be expected in the absence of disease, inter-individual variation is likely with strenuous physical exercise.

It seems important to discuss the possible consequences of exercise and sickle cell trait which are not currently clear in the literature. Case-control studies have found an increased prevalence of sudden death on exertion, often called "exercise collapse associated with sickle trait" however a direct causal relationship has not been confirmed [29]. It has been suggested that a combination of factors [30] such as high altitude or severe dehydration combined with intense physical activity could cause red blood cells to become deformed or sickled and cause complications such as rhabdomyolysis. This may be due to lower capillary density and tortuosity, reduced small vessels and a higher percentage of broader micro vessels [31]. Microvascular remodeling may be a compensatory mechanism to facilitate normal blood flow [29] which may be present in this patient considering the raised but not excessive elevation of CK following a marathon (data not presented). SCT may not be a completely benign carrier state nor a true disease entity but rather a potential risk factor which could be monitored. Maintaining physical fitness may be a protective agent by decreasing the endothelial activation through improvements in nitric oxide and antioxidant availability [32, 33]. Precautions would be the same as for exercise with MG i.e.; adequate hydration, control heat exposure and monitor exertion intensity [34].

This case report demonstrates that despite being in the first 2 years of MG disease onset in which exacerbations and instability are most common, and despite regular intense endurance running, the MG has remained stable. A growing body of literature suggests that regular physical exercise may lead to anti-inflammatory effects in chronic auto-immune diseases. The immunomodulatory role of exercise could be considered in this case study. However, further studies regarding the specific effects of exercise on disease activity in MG are necessary to confirm these speculations.

\section{Patient perspective}

"I am myasthenic and marathonian, running allows me to take time for myself, to evacuate stress and to think. It's a real therapy".

\section{Abbreviations}

6MWD: Six-minute walk distance; AChR: Acetycholine receptor; b.i.d: Twice a day; CN: Cranial nerve; EMG: Electromyogram; IL-6: Interleukin-6; MG: Autoimmune myasthenia gravis; MG-ADL: Myasthenia Gravis Activites of Daily Living score; MGFA: Myasthenia Gravis Foundation of America disease classification; MGQOL-15-F: Myasthenia Gravis 15 item French Quality Of Life scale; MIP/MEP: Maximal inspiratory pressure/Maximal expiratory pressure; MMS: Myasthenic Muscle Score; SCT: Sickle cell trait; ti.d: Three times a day; TNF-a: Tumour Necrosis Factor alpha; UL: Upper limb; WHO-QOL Bref: World Health Organisation Quality Of Life short questionnaire

\section{Acknowledgements \\ The authors would like to thank the patient for accepting publication of her case. Thank you to Aurelie Canal for her assistance in data collection and presentation.}

Funding

No specific funding was provided for this case report. 


\section{Availability of data and materials}

All data analysed in this case report are included in this published article.

\section{Disclaimer}

This is a case report and is by no means a reflection of common practice. Several factors may have contributed to disease stability in this subject.

\section{Authors' contributions}

SB contributed to the concept, drafting and reporting of the case. MT, BE and SB acquired clinical data and revised the manuscript. TS, PP, JY, contributed to reporting and revising the manuscript. All authors read and approved the final manuscript.

\section{Ethics approval and consent to participate}

Not applicable.

\section{Consent for publication}

Full written consent was provided by the patient for publication.

\section{Competing interests}

The authors declare that they have no competing interests.

\section{Publisher's Note}

Springer Nature remains neutral with regard to jurisdictional claims in published maps and institutional affiliations.

\section{Author details}

'Institute of Myology, GH Pitié-Salpêtrière (AP-HP), Bd de l'Hôpital, 75651 Paris Cedex 13, France. ${ }^{2}$ Bioingénierie, Tissus et Neuroplasticité, EA 7377 Université Paris-Est Créteil Faculté de Médecine, 8 rue Jean Sarrail, 94010 Créteil, France. ${ }^{3}$ Unité de Recherche Clinique Paris Île- de- France Ouest (URC PIFO), Raymond Poincaré Hospital, AP-HP, Garches, France. ${ }^{4}$ Medical and Surgical Neurointensive Care Centre, Hospital Sainte Anne, Paris, France. ${ }^{5}$ Laboratory of human histopathology and animal models, Institute Pasteur, Paris, France. ${ }^{6}$ Université Paris Descartes, Paris, France. ${ }^{7}$ Department of Neurology, CHUV, Rue du Bugnon, 461011 Lausanne, Switzerland.

\section{Received: 26 June 2018 Accepted: 12 September 2018}

\section{Published online: 18 September 2018}

\section{References}

1. Phillips WD, Vincent A. Pathogenesis of myasthenia gravis: update on disease types, models, and mechanisms. F1000Res. 2016;5(F1000 Faculty Rev):1513.

2. Paul RH, Nash JM, Cohen RA, Gilchrist JM, Goldstein JM. Quality of life and well-being of patients with myasthenia gravis. Muscle Nerve. 2001;24:512-6.

3. Scheer BV, Valero-Burgos E, Costa R. Myasthenia gravis and endurance exercise. Am J Phys Med Rehabil. 2012;91:725-7.

4. Stout JR, Eckerson JM, May E, Coulter C, Bradley-Popovich GE. Effects of resistance exercise and creatine supplementation on myasthenia gravis: a case study. Med Sci Sports Exerc. 2001:33:869-72.

5. Leddy JJ, Chutkow JG. Myasthenia gravis in a collegiate football player. Med Sci Sports Exerc. 2000:32:1975-9.

6. Rahbek MA, Mikkelsen EE, Overgaard K, Vinge L, Andersen H, Dalgas U. Exercise in myasthenia gravis: a feasibility study of aerobic and resistance training. Muscle Nerve. 2017;56:700-9.

7. Westerberg E, Molin CJ, Lindblad I, Emtner M, Punga AR. Physical exercise in myasthenia gravis is safe and improves neuromuscular parameters and physical performance-based measures: a pilot study. Muscle Nerve. 2017;56: 207-14.

8. Westerberg E, Molin CJ, Sporndly Nees S, Widenfalk J, Punga AR. The impact of physical exercise on neuromuscular function in myasthenia gravis patients: a single-subject design study. Medicine. 2018;97:e11510.

9. Lohi EL, Lindberg C, Andersen O. Physical training effects in myasthenia gravis. Arch Phys Med Rehabil. 1993;74:1178-80.

10. Cass S. Myasthenia gravis and sports participation. Curr Sports Med Rep. 2013;12:18-21

11. Sussman J, Farrugia ME, Maddison P, Hill M, Leite MI, Hilton-Jones D. Myasthenia gravis: Association of British Neurologists' management guidelines. Pract Neurol. 2015;15:199-206.
12. Sanders DB, Wolfe Gl, Narayanaswami P. Developing treatment guidelines for myasthenia gravis. Ann N Y Acad Sci. 2018;1412:95-101.

13. Skevington SM, Lotfy M, O'Connell KA. The World Health Organization's WHOQOL-BREF quality of life assessment: psychometric properties and results of the international field trial. A report from the WHOQOL group. Qual Life Res. 2004;13:299-310.

14. Hawtho rne G, Herrman H, Murphy B. Interpreting the WHOQOL-BREF: preliminary population norms and effect sizes. Soc Indic Res. 2006;77:37-59.

15. Barros ES, Nascimento DC, Prestes J, Nobrega OT, Cordova C, Sousa F Boullosa DA. Acute and chronic effects of endurance running on inflammatory markers: a systematic review. Front Physiol. 2017:8:779.

16. Niemela M, Kangastupa $P$, Niemela $O$, Bloigu $R$, Juvonen T. Acute changes in inflammatory biomarker levels in recreational runners participating in a Marathon or half-Marathon. Sports Med Open. 2016;2:21.

17. Pedersen BK. Muscle as a secretory organ. Compr Physiol. 2013:3:1337-62.

18. Perandini LA, Sales-de-Oliveira D, Mello S, Camara NO, Benatti FB, Lima FR, Borba E, Bonfa E, Roschel H, Sa-Pinto AL, Gualano B. Inflammatory cytokine kinetics to single bouts of acute moderate and intense aerobic exercise in women with active and inactive systemic lupus erythematosus. Exerc Immunol Rev. 2015;21:174-85.

19. Uzawa A, Kawaguchi N, Himuro K, Kanai T, Kuwabara S. Serum cytokine and chemokine profiles in patients with myasthenia gravis. Clin Exp Immunol. 2014;176:232-7

20. Uzawa A, Kanai T, Kawaguchi N, Oda F, Himuro K, Kuwabara S. Changes in inflammatory cytokine networks in myasthenia gravis. Sci Rep. 2016;6:25886.

21. Roche JC, Capablo JL, Larrad L, Gervas-Arruga J, Ara JR, Sanchez A, Alarcia R. Increased serum interleukin-17 levels in patients with myasthenia gravis. Muscle Nerve. 2011:44:278-80.

22. Li Y, Rauniyar VK, Yin WF, Hu B, Ouyang S, Xiao B, Yang H. Serum IL-21 levels decrease with glucocorticoid treatment in myasthenia gravis. Neurol Sci. 2014:35:29-34.

23. Molin CJ, Westerberg E, Punga AR. Profile of upregulated inflammatory proteins in sera of myasthenia gravis patients. Sci Rep. 2017:7:39716.

24. Punga $T$, Le Panse $R$, Andersson $M$, Truffault $F$, Berrih-Aknin S, Punga AR. Circulating miRNAs in myasthenia gravis: miR-150-5p as a new potentia biomarker. Ann Clin Transl Neurol. 2014;1:49-58.

25. Punga AR, Punga T. Circulating microRNAs as potential biomarkers in myasthenia gravis patients. Ann N Y Acad Sci. 2018;1412:33-40.

26. Munoz-Canoves P, Scheele C, Pedersen BK, Serrano AL. Interleukin-6 myokine signaling in skeletal muscle: a double-edged sword? FEBS J. 2013; 280:4131-48.

27. Belizario JE, Fontes-Oliveira CC, Borges JP, Kashiabara JA, Vannier E. Skeletal muscle wasting and renewal: a pivotal role of myokine IL-6. Springerplus. 2016:5:619.

28. Maurer M, Bougoin S, Feferman T, Frenkian M, Bismuth J, Mouly V, Clairac G, Tzartos S, Fadel E, Eymard B, et al. IL-6 and Akt are involved in muscular pathogenesis in myasthenia gravis. Acta Neuropathol Commun. 2015;3:1.

29. O'Connor FG, Bergeron MF, Cantrell J, Connes P, Harmon KG, Ivy E, Kark J, Klossner D, Lisman P, Meyers BK, et al. ACSM and CHAMP summit on sickle cell trait: mitigating risks for warfighters and athletes. Med Sci Sports Exerc. 2012:44:2045-56.

30. Quattrone RD, Eichner ER, Beutler A, Adams WB, O'Connor FG. Exercise collapse associated with sickle cell trait (ECAST): case report and literature review. Curr Sports Med Rep. 2015;14:110-6.

31. Vincent L, Feasson L, Oyono-Enguelle S, Banimbek V, Denis C, Guarneri C, Aufradet E, Monchanin G, Martin C, Gozal D, et al. Remodeling of skeletal muscle microvasculature in sickle cell trait and alpha-thalassemia. Am J Physiol Heart Circ Physiol. 2010;298:H375-84.

32. Chirico EN, Faes C, Connes P, Canet-Soulas E, Martin C, Pialoux V. Role of exercise-induced oxidative stress in sickle cell trait and disease. Sports Med. 2016:46:629-39.

33. Aufradet $E$, Monchanin $G$, Oyonno-Engelle $S$, Feasson L, Messonnier L, Francina A, Bezin L, Serpero LD, Gozal D, Dodogba M, et al. Habitual physical activity and endothelial activation in sickle cell trait carriers. Med Sci Sports Exerc. 2010;42:1987-94.

34. Eichner ER. Sickle cell considerations in athletes. Clin Sports Med. 2011:30: $537-49$ 